\title{
La magnétohydrodynamique \\ ou \\ ces fluides qui conduisent l'électricité
}

\author{
René Moreau \\ Professeur à l'INPG/ENSHMG, Laboratoire MADYLAM \\ Membre de l'Académie des Sciences
}

L'Univers est essentiellement constitué de matière fluide conductrice de l'électricité. Cela ne concerne pas que les étoiles, gigantesques boules de gaz ionisés, ou d'autres objets astrophysiques, à vrai dire bien éloignés de notre sphère d'activités humaines. Cela concerne aussi la Terre, énorme boule de métal, en grande partie liquide, déjà beaucoup plus centrée dans nos préoccupations, et dont la surface est largement occupée par de l'eau salée, elle aussi conductrice de l'électricité. Mais, au cœur même de notre civilisation industrielle, où la grande majorité des objets manufacturés sont constitués de métaux qui, à un certain stade de leur élaboration, passent par l'état liquide, on rencontre souvent des fluides conducteurs de l'électricité. Le tableau 1 montre des valeurs typiques des conductivités électriques et des masses volumiques des fluides les plus courants. On notera que les métaux fondus sont 10000 fois

Tableau 1. - Conductivités électriques typiques.

\begin{tabular}{|l|c|}
\hline \multicolumn{1}{|c|}{ Matériaux } & $\sigma\left(\Omega^{-1} \mathrm{~m}^{-1}\right)$ \\
\hline Eau distillée & $\approx 10^{-4}$ \\
Electrolytes faibles & $10^{-4}$ à $10^{-2}$ \\
Electrolytes forts & $10^{-2}$ à $10^{2}$ \\
- Eau $+\mathrm{NaCl}$ à $25 \%\left(20^{\circ} \mathrm{C}\right)$ & 21,6 \\
- $\mathrm{H}_{2} \mathrm{SO}_{4}$ pur $\left(20^{\circ} \mathrm{C}\right)$ & 73,6 \\
Verre fondu $\left(1400^{\circ} \mathrm{C}\right)$ & $10^{2}$ \\
Plasmas " froids" $\left(T \approx 10^{4} \mathrm{~K}\right)$ & $\approx 10^{3}$ \\
Plasmas " chauds" $\left(T \approx 10^{6} \mathrm{~K}\right)$ & $\approx 10^{6}$ \\
Gaz totalement ionisés & $\approx 10^{7} \cdot T^{3 / 2}$ \\
Métaux liquides & $10^{6}$ à $10^{7}$ \\
- Acier (1500 $\left.{ }^{\circ} \mathrm{C}\right)$ & $0,7.10^{6}$ \\
- Mercure $\left(20^{\circ} \mathrm{C}\right)$ & $10^{6}$ \\
- Aluminium $\left(700^{\circ} \mathrm{C}\right)$ & $5.10^{6}$ \\
- Sodium $\left(400^{\circ} \mathrm{C}\right)$ & $6.10^{6}$ \\
Métaux solides & $10^{6}$ à $10^{8}$ \\
- Acier $\left(20^{\circ} \mathrm{C}\right)$ & $\approx 10^{6}$ \\
- Sodium $\left(20^{\circ} \mathrm{C}\right)$ & $\approx 10^{7}$ \\
- Cuivre $\left(20^{\circ} \mathrm{C}\right)$ & $6.10^{7}$ \\
\hline
\end{tabular}

plus conducteurs que les électrolytes et 60 fois moins que le cuivre à l'état solide.

La mécanique des fluides a donc dû s'ouvrir à ces fluides conducteurs. Et, comme on le verra, c'est lorsqu'un champ magnétique est présent, qu'il apparaisse spontanément comme c'est le cas à l'échelle astrophysique ou géophysique, ou qu'on l'applique artificiellement avec des aimants, comme c'est le cas à l'échelle du laboratoire, que cette mécanique devient tout à fait originale, voire spectaculaire. Dans ce qui suit, on s'attache à dégager quelques propriétés remarquables de portée très générale, et, pour les illustrer, on fait le choix de mettre davantage l'accent sur des phénomènes de MHD observables au laboratoire (avec des métaux liquides comme le mercure).

\section{L'équation de l'induction et le nombre de Reynolds magnétique}

Le référentiel le mieux adapté à la description du mouvement d'un fluide est celui du laboratoire dans lequel la particule fluide se déplace à la vitesse $\vec{u}$. La loi d'Ohm (qui s'écrirait $\vec{j}^{\prime}=\sigma \vec{E}^{\prime}$ dans le référentiel lié à la particule) s'écrit alors

$$
\vec{j}=\sigma(\vec{E}+\vec{u} \wedge \vec{B}) .
$$

où

$$
\vec{E}=-\vec{\nabla} \varphi-\frac{\partial \vec{A}}{\partial t}
$$

désigne le champ électrique, $\vec{j}$ la densité de courant, $\varphi$ le potentiel électrique, $\overrightarrow{\mathrm{A}}$ le potentiel vecteur $(\vec{B}=\overrightarrow{\operatorname{rot}} \vec{A})$ et $\sigma$ la conductivité électrique. Compte tenu des équations de Maxwell qui permettent d'exprimer toutes ces grandeurs en fonction de $\vec{B}$ (voir [1]), il est commode de considérer $\vec{B}$ comme la grandeur électromagnétique fondamentale. Et l'équation d'évolution de $\vec{B}$, en général appelée l'équation de l'induction, qui n'est autre que le rotationnel de (1), s'écrit ( $\mu$ désigne la perméabilité magnétique) :

$$
\frac{\partial \vec{B}}{\partial t}=\overrightarrow{\operatorname{rot}}(\vec{u} \wedge \vec{B})+\frac{1}{\mu \sigma} \nabla^{2} \vec{B} .
$$


Soient $U$ et $L$ des échelles de vitesse et de longueur caractéristiques du phénomène. On appelle le nombre de Reynolds magnétique la quantité sans dimension

$$
R m=\mu \sigma U L
$$

qui représente le rapport du premier au second terme du deuxième membre de l'équation (3).

Lorsque $R m \ll 1$, l'équation (3) peut, au premier ordre, être réduite à sa forme dans les milieux au repos. Et la perturbation du champ magnétique $\vec{B}$, que l'on notera $\vec{b}$. est de l'ordre de $B R m$. Au contraire, lorsque $R m \geqslant 1$, c'est le dernier terme du second membre qui devient négligeable et, dans la limite $R m \rightarrow \infty$, l'équation de l'induction se ramène à:

$$
\frac{\partial \vec{B}}{\partial t}=\overrightarrow{\operatorname{rot}}(\vec{u} \wedge \vec{B}) .
$$

Celle-ci est bien connue en hydrodynamique : elle est analogue à l'équation du tourbillon en fluide parfait (ou lorsque le nombre de Reynolds ordinaire devient infini). On sait bien quelles en sont les conséquences: comme le tourbillon $(\overrightarrow{\text { rot }} \vec{u})$ en fluides ordinaires, en MHD le champ magnétique est gelé dans la matière. Autrement dit, il est extrêmement difficile de faire pénétrer le champ magnétique dans la matière. La figure 1 montre bien que la pénétration du champ magnétique dans un cylindre tournant (rayon $R$. vitesse angulaire $\Omega$ ) est excellente si $R m<1$ et de plus en plus faible si $R m>1$ et continue à crôtre.

Dans les métaux liquides à l'échelle du laboratoire, avec les estimations suivantes: $(\mu \sigma)^{-1}=1 \mathrm{~m}^{2} \mathrm{~s}^{-1}$, $U=10^{-1} \mathrm{~m} \mathrm{~s} s^{-}, L=10^{-1} \mathrm{~m}$, on peut situer $R m$ aux alentours de $10^{2}$. L'évolution du champ magnétique est alors dominée par la diffusion.

Aux échelles géophysiques $\left(R m=10^{4}\right)$ ou astrophysiques $\left(R m \geqslant 10^{6}\right)$, la convection devient prépondérante et l'approximation du champ gelé est bien justifiée. A titre d'exemple, cette propriété est à la base de l'explication des taches solaires, régions d'où la matière a été emportée par les tubes de flux magnétique dans leur mouvement.

Le domaine intermédiaire, où $R m=10$ à $10^{2}$. est particulièrement intéressant, car il est marqué par un phénomène spectaculaire : l'effet dynamo, ou bien l'auto-excitation spontanée du champ magnétique, et son entretien à partir du champ de vitesse, malgré les pertes par dissipation ohmique. La configuration la plus simple, pour illustrer l'effet dynamo, est celle de la figure 2 , où le domaine fluide en mouvement est simulé par un disque tournant en bloc à la vitesse angulaire $\Omega$. Une spire enroulée autour du disque et de son axe dans le sens indiqué sur la figure (ce sens est un ingrédient essentiel) permet à un courant électrique d'intensité $l$, s'il en apparait un, de se refermer dans le circuit ainsi réalisé. Le régime non magnétique $(B=0, I=0, \Omega=C / k$. où $C$ désigne le couple appliqué et $k$ le coefficient de frottement) est possible. Imaginons maintenant une perturbation électromagnétique $(I \neq 0)$ comme indiqué sur la figure. Le champ électromoteur $\vec{u} \wedge \vec{B}$ présent dans le disque a bien le signe nécessaire (à cause du sens du bobinage) pour engendrer une force électro-motrice capable de vaincre les chutes de tension ohmiques dans le circuit. Cet état magnétique apparaît donc lui aussi possible. On montre qu'il existe un couple critique $C^{*}$ et une vitesse critique $\Omega^{*}$ tels que : - si $C<C^{*}$, la seule solution stable des équations du problème est la solution non magnétique,
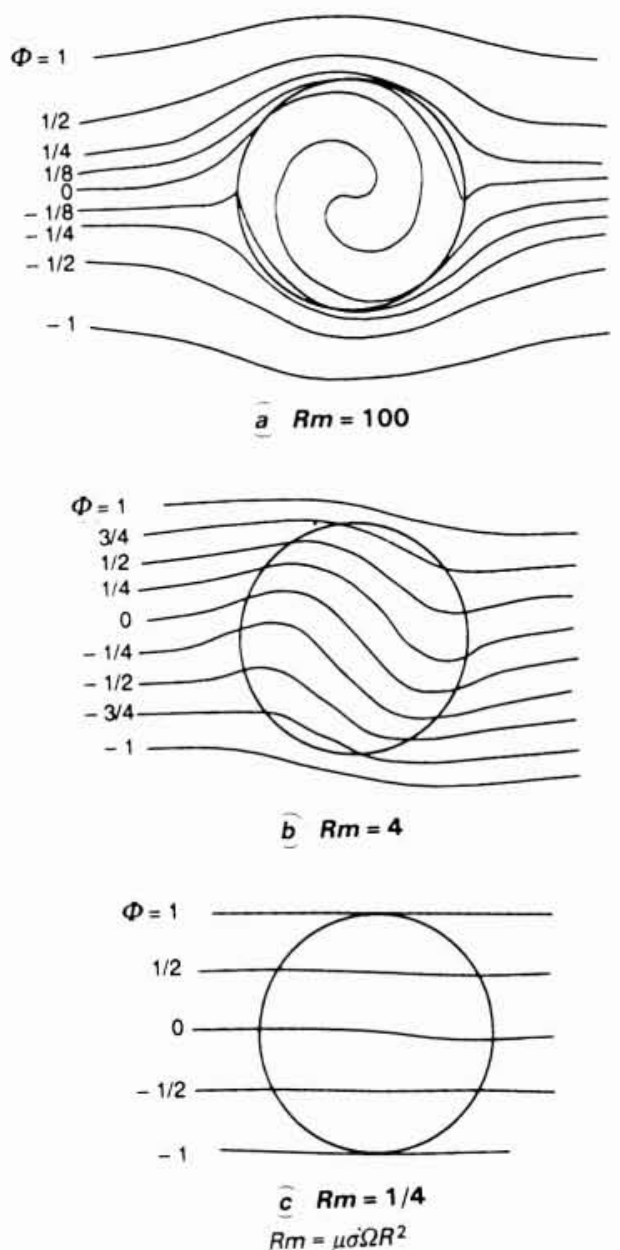

1. Déformation des lignes de flux magnétique par un cylindre conducteur de rayon $R$ tournant à la vitesse $\Omega$ pour diverses valeurs du nombre de Reynolds magnétique $\mathbf{R m}$. Le champ magnétique est uniforme à l'infini. Le milieu extérieur au cylindre est isolant.

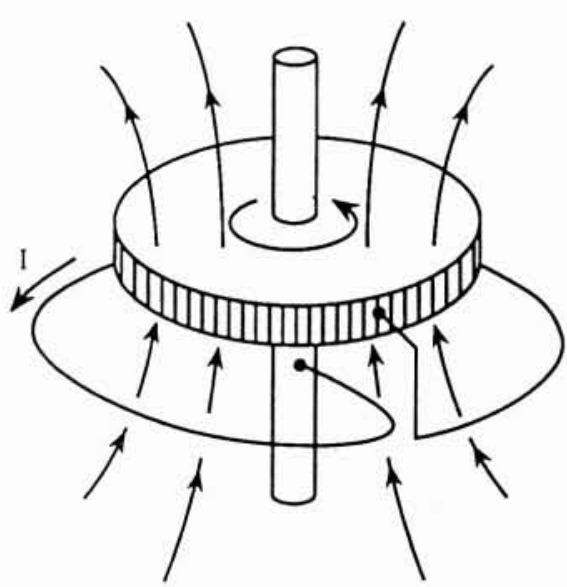

2. Schéma illustrant le fonctionnement d'une dynamo homopolaire. 
- si $C>C^{*}$, la seule solution stable des équations est la solution magnétique, caractérisée par les propriétés (en régime permanent) :

$$
I=\left(\frac{C-C^{*}}{M}\right)^{1 / 2}, \quad \Omega=\Omega^{*}
$$

où $M$ est la mutuelle inductance entre le disque et la spire (fig. 3).
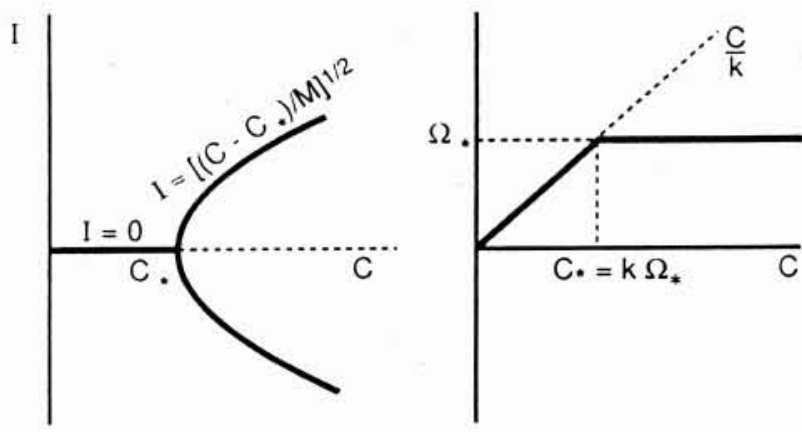

3. Courbes caractéristiques du fonctionnement d'une dynamo homopolaire en régime permanent.

On notera les trois critères nécessaires à l'effet dynamo : - un défaut de symétrie (ici lié au sens du bobinage), - un cisaillement (ici localisé au niveau des contacts disquespire),

- et un couple suffisant, ou une vitesse suffisante, ou encore un nombre de Reynolds magnétique suffisant (de 10 à $10^{2}$ suivant la configuration).

Et l'on remarquera aussi les propriétés curieuses du régime magnétique :

- la vitesse est bloquée $\left(\Omega^{*}=R / M\right)$ et ne dépend plus du couple, sa valeur est fixée par les lois de l'électromagnétisme,

- le courant électrique croît comme $\left(C-C^{*}\right)^{1 / 2}$ lorsque l'on fournit de plus en plus de puissance au système et ce sont les lois de la mécanique qui fixent sa valeur.

C'est cette bifurcation du régime non magnétique vers un régime magnétique qui explique la présence et l'entretien du champ magnétique terrestre malgré la dissipation par effet Joule. Celle-ci, à elle seule, éteindrait le champ magnétique terrestre en $10^{4}$ années. Or les études du paléomagnétisme révèlent que, depuis $10^{8}$ années, la Terre possède un champ magnétique. Celui-ci n'est d'ailleurs pas stationnaire : son évolution est une sorte de signature de la variation des mouvements de convection au centre de la Terre (rendus non symétriques par la force de Coriolis). A titre d'exemple de cette évolution, les pôles s'inversent environ toutes les $10^{5}$ années.

\section{L'équation du mouvement et le nombre de Hartmann}

En présence d'un champ magnétique $\vec{B}$, chaque unité de volume parcourue par une densité de courant $\vec{j}$ est soumise à une force de Laplace $\vec{j} \wedge \vec{B}$. L'équation de Navier-Stokes, qui gouverne le mouvement du fluide, s'écrit donc :

$$
\rho \frac{\mathrm{d} \vec{u}}{\mathrm{~d} t}=-\vec{\nabla} p+\rho v \nabla^{2} \vec{u}+\rho \vec{g}+\vec{j} \wedge \vec{B}
$$

( $\rho$ désigne la masse volumique, $p$ la pression, $g$ la gravité, $v$ la viscosité cinématique). Dans les situations les plus courantes de la mécanique des fluides classiques, les termes d'inertie, de pression et de gravité sont du même ordre, et le terme de viscosité est beaucoup plus petit qu'eux. Le si célèbre nombre de Reynolds $(\mathrm{Re}=U L / v)$ mesure justement le rapport des termes d'inertie au terme de viscosité. En MHD, avec une force nouvelle, on est amené à introduire un nouveau nombre sans dimension pour mesurer l'influence relative de celle-ci. On définit le nombre de Hartmann

$$
H a=B L(\sigma / \rho \nu)^{1 / 2},
$$

dont le carré représente le rapport de la force de Laplace aux forces de viscosité. Et la combinaison

$$
N=\sigma B^{2} L / \rho U=H a^{2} / \operatorname{Re},
$$

qui mesure le rapport de la force Laplace à la force d'inertie, est appelée paramètre d'interaction.

Dans les métaux liquides à l'échelle du laboratoire, avec un champ magnétique de l'ordre de 1 Tesla, facile à produire avec des aimants, on obtient aisément des nombres de Hartmann de l'ordre de $10^{2}$ à $10^{3}$ et des paramètres d'interaction de l'ordre de 1 . Ceci veut dire que l'effet mécanique du champ magnétique appliqué peut être prépondérant, même si la réaction magnétique du champ de vitesse demeure faible (puisque $R m \ll 1$ ).

\section{L'écoulement de Hartmann}

L'exemple le plus simple d'écoulement MHD est l'analogue de l'écoulement de Poiseuille: régime établi dans une conduite très longue engendré par un gradient de pression uniforme. Supposons la conduite placée dans l'entrefer d'un aimant (fig. 4) et, pour nous permettre de mettre en évidence

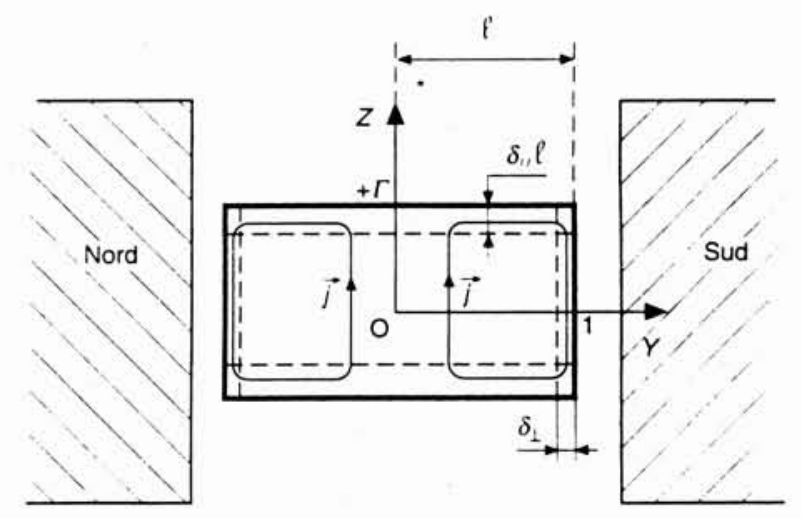

4. Configuration typique de l'écoulement de Hartmann. Les parois de la conduite sont, soit perpendiculaires, soit parallèles au champ magnétique. Des lignes de courant électrique typiques sont tracées en supposant les parois isolantes. Les symboles $\delta_{\perp}$ et $\delta_{\|}$désignent respectivement l'épaisseur de la couche de Hartmann et l'épaisseur des couches parallèles au champ magnétique. 
I'influence de l'orientation de la paroi par rapport au champ magnétique, supposons la conduite rectangulaire, avec deux côtés perpendiculaires à $\vec{B}$ et deux côtés parallèles à $\vec{B}$.

Dans la zone centrale de la conduite, le champ électromoteur $\vec{u} \wedge \vec{B}$ pousse les ions positifs dans la direction $O z$. Mais, sur l'une ou l'autre des faces de la paroi, il existe une frontière isolante où le courant électrique ne peut pas passer. Conformément à la loi d'Ohm (1), un potentiel électrique s'installe, maximum en haut de la conduite et minimum en bas, de telle sorte que le champ électrique $\vec{E}=-\vec{\nabla}$ s'oppose au champ électromoteur. Au centre, où la vitesse est la plus grande, c'est le champ électromoteur qui l'emporte sur le champ électrique, mais près des parois où la vitesse est la plus faible, c'est le champ électrique qui l'emporte.

Supposons d'abord la paroi isolante (cas de la fig. 4). Ce courant de retour est localisé dans une couche limite, appelée couche de Hartmann. La valeur moyenne de la densité de courant $j$. est alors nulle. En conséquence, la valeur moyenne de la force de Laplace est nulle, elle aussi. Le freinage de l'écoulement par le champ magnétique est dans ce cas modéré. On constate surtout une forte modification du profil de vitesse, puisque la force de Laplace est négative au centre et positive dans la couche de Hartmann (fig. $5 a$ ).

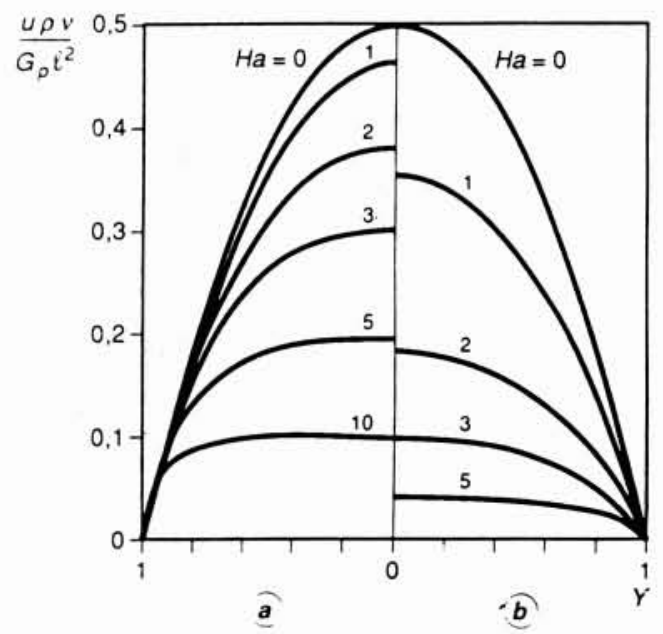

5. Distribution de vitesse $\boldsymbol{u}(\boldsymbol{Y})$ dans le plan de symétrie $Z=0$ de la figure 4 ( $a$ : si les parois sont isolantes, $b$ : si les parois sont parfaitement conductrices).

Supposons au contraire la paroi parfaitement conductrice. Tout le courant de retour transite alors dans celle-ci, dont la résistance est nulle. La densité de courant $j_{\text {: }}$ est positive même dans la couche de Hartmann. La valeur moyenne de $j$ : d'un bord à l'autre de la conduite n'est plus nulle et la résistance à l'avancement du fluide est beaucoup plus grande que dans le cas d'une paroi isolante. La théorie de cet écoulement montre que la vitesse au centre est $\mathrm{Ha}$ fois plus grande avec des parois très conductrices qu'avec des parois isolantes. Pour résumer, retenons que la distribution de vitesse le long de $O y$ présente un plateau central. La valeur de la vitesse maximale est $(-\mathrm{d} p / \mathrm{d} x) \rho / \sigma B^{2}$ si la paroi est parfaitement conductrice, et $\mathrm{Ha}$ fois plus grande si la paroi est isolante. Il va de soi que, si la conductance de la paroi est finie, le résultat est intermédiaire entre ces deux extrêmes. L'épaisseur de la couche de Hartmann est dans tous les cas la même $(\rho v / \sigma)^{1 / 2} / B$; on remarquera qu'elle ne dépend pas de la largeur de la conduite.

Au voisinage des parois parallèles au champ magnétique les couches limites sont d'un autre type. Pour illustrer leur principale caractéristique, limitons-nous au cas un peu singulier où ces parois parallèles au champ magnétique sont isolantes et où les parois perpendiculaires sont très conductrices. Le courant ne pouvant pas traverser les parois parallèles à $\vec{B}$, dans leur voisinage $\vec{j}$ devient parallèle à celles-ci et au champ magnétique, de sorte que, dans la couche limite, la force de Laplace s'effondre. Cette propriété conduit à une distribution de vitesse tout à fait curieuse, puisque le fluide est fortement freiné au centre de la conduite (vitesse proportionnelle à $B^{-2}$ ). On observe dans les couches parallèles à $\vec{B}$ des sortes de jets assez rapides illustrés sur la figure 6 (la vitesse $y$ est $\mathrm{Ha}$ fois plus grande qu'au centre) et assez minces (leur largeur est de l'ordre de $l . \mathrm{Ha}^{-1 / 2}$ ).
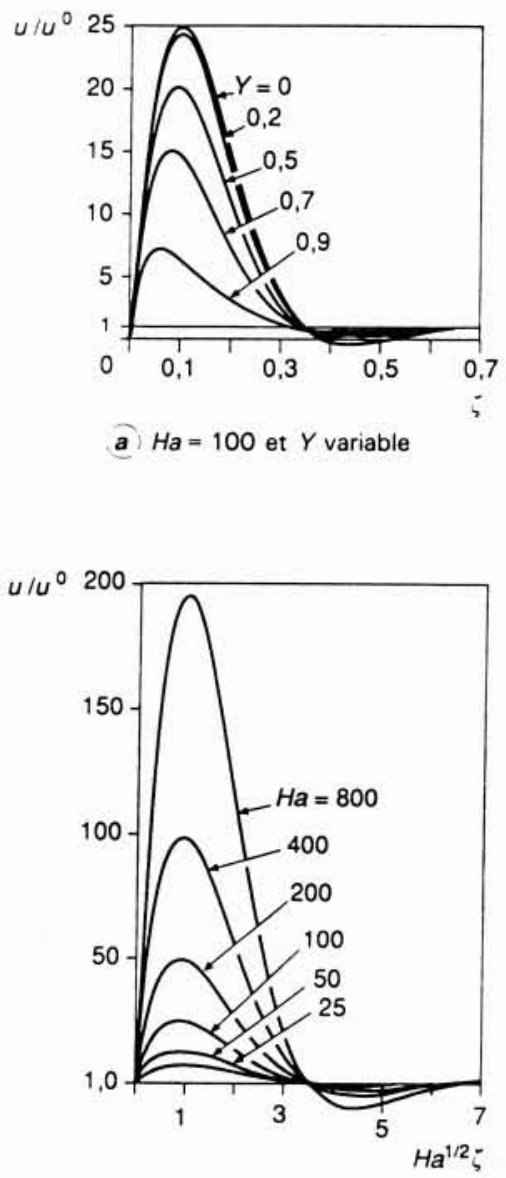

b $Y=0$ et $\mathrm{Ha}$ variable

6. Distribution de vitesse $u(Z)$ à travers une couche parallèle de la conduite rectangulaire de la figure 4 dans le cas particulier où les parois parallèles au champ magnétique sont isolantes et les parois perpendiculaires parfaitement conductrices. La variable $\zeta$ désigne la coordonnée sans dimension $\mathrm{ZH}_{a}^{-1 / 2}$. 


\section{Les ondes d'Alfven}

Même en supposant le fluide absolument incompressible, ce qui élimine l'acoustique, des ondes peuvent se propager dans les fluides conducteurs de l'électricité soumis à un champ magnétique. Celles-ci, découvertes par Alfven [2], proviennent précisément du couplage entre les équations de Maxwell et les équations de Navier-Stokes, elles représentent l'un des phénomènes les plus caractéristiques de la MHD. On verra plus loin que leurs propriétés sont indispensables à l'interprétation de nombreuses situations parmi les plus curieuses.

Nous nous limiterons ici à une brève description du mécanisme de propagation de ces ondes, renvoyant le lecteur à des ouvrages plus complets [1], [2], pour une analyse de leur théorie. Supposons d'abord le fluide assez bon conducteur et assez peu visqueux pour pouvoir négliger totalement les effets dissipatifs. Imaginons ce fluide illimité et au repos en présence d'un champ magnétique uniforme $\vec{B}=\left(B_{0}\right.$, $0,0)$. Créons soudainement une perturbation en déplaçant la tranche fluide $A B C D$ perpendiculairement au plan de la figure 7 avec une vitesse $\vec{u}=(0, v, 0)$. Le champ électromoteur $\vec{u} \wedge \vec{B}=\left(0,0,-v B_{0}\right)$ pousse les ions dans la direction opposée à $O z$, et un champ électrique doit apparaître, de telle façon que les lignes de courant électrique puissent se refermer de part et d'autre de $A B C D$. Dans cette tranche $A B C D$, le champ électromoteur l'emporte sur le champ électrique et le courant électrique circule bien dans la direction opposée à $O z$. Mais à l'extérieur de $A B C D$, le courant électrique circule dans le sens de $O z$ sous l'effet du champ électrique. Comme on peut le voir sur la figure 7 , les lignes de courant électrique forment deux spires de sens opposés qui induisent une perturbation $\vec{b}$ du champ magnétique $\vec{B}$, et celle-ci est colinéaire à la vitesse $\vec{u}$. La force de Laplace, qui se réduit à $\vec{j} \wedge \vec{B}=\left(0, j_{z} B_{0}, 0\right)$, est dirigée suivant $O y$ et a le même signe que $j_{z}$. On voit qu'elle freine la tranche $A B C D$, initialement mise en mouvement. Ceci est bien conforme à la loi de l'induction : les effets électromagnétiques s'opposent toujours au mécanisme qui leur a donné naissance. Mais cette force de Laplace a aussi pour effet d'accélérer et de mettre en mouvement les tranches voisines $B B^{\prime} C^{\prime} C$ et $A^{\prime} A D D^{\prime}$. C'est ainsi qu'elle propage la perturbation initiale. On peut bien sûr prolonger le raisonnement et se convaincre qu'après un certain temps deux portions du fluide, $M N P Q$ d'un côté, $M^{\prime} N^{\prime} P^{\prime} Q^{\prime}$ de l'autre, seront en mouvement à leur tour, avec la vitesse $\vec{u}$ et la perturbation magnétique $\vec{b}$ dirigées suivant $O y$ (alors que la propagation se fait dans la direction $O x$ ).

La théorie de ces ondes permet de bien établir leurs propriétés, seulement esquissées ci-dessus. La plus impor-

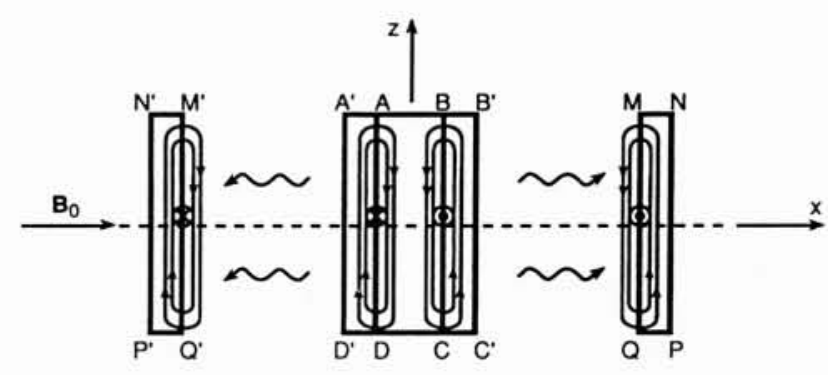

7. Illustration du mécanisme de propagation des ondes d'Alfven dans les directions $\pm \vec{B}_{0}$. tante est sûrement le fait que la vitesse de groupe, appelée vitesse d'Alfven, est dirigée dans les deux directions $\pm \vec{B}$ et a pour module $B_{0}(\mu \rho)^{-1 / 2}\left(\right.$ si $B_{0} \cong 10^{-1} \mathrm{~T}$, celle-ci est de l'ordre de $1 \mathrm{~m} \mathrm{~s}^{-1}$ ). A la différence des ondes sonores, ces ondes d'Alfven sont donc tout à fait anisotropes. Elles transportent bien sûr leur énergie (cinétique et magnétique). Et, si elles disposent d'une durée suffisante, elles tendent à uniformiser les distributions des diverses grandeurs dans la direction du champ magnétique appliqué.

La première expérience de laboratoire qui ait bien mis en évidence cette conséquence des ondes d'Alfven a été réalisée par Lehnert [3]. Comme le montre la figure 8, le moment angulaire des couches fluides situées au-dessus de l'anneau tournant est uniforme, jusqu'à la surface libre, et égal à celui de l'anneau de cuivre tournant. De même, les couches de mercure situées au-dessus des disques de cuivre fixes demeurent immobiles. Sans les ondes d'Alfven, qui installent cette sorte de rigidité verticale, on observerait sur la surface libre une image beaucoup plus diffuse de la rotation de l'anneau.

En réalité, les fluides ont une résistivité et une viscosité finies, et ces effets dissipatifs atténuent les ondes d'Alfven. Dans les métaux liquides la dissipation ohmique est de très loin la plus importante ; elle conduit à un temps caractéristique pour amortir une perturbation de longueur $\lambda$ égal à $\mu \sigma \lambda^{2}$. Avec $\lambda=1 \mathrm{~m}$, ce temps est de l'ordre de $1 \mathrm{~s}$. Puisque la vitesse d'Alfven est de l'ordre de $1 \mathrm{~m} \mathrm{~s}^{-1}$, cela veut dire que dans les métaux liquides, les ondes d'Alfven sont atténuées après des distances de l'ordre du mètre. L'expé-

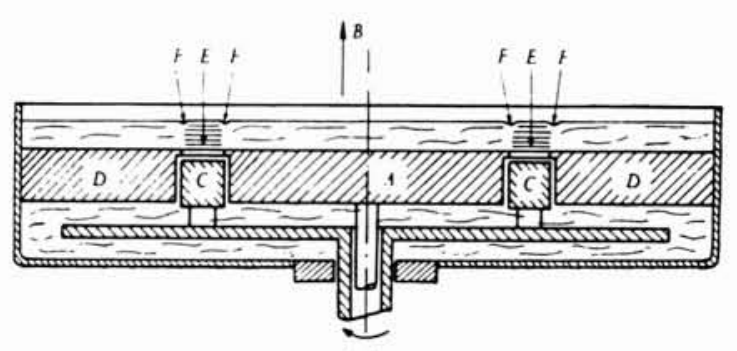

(a)

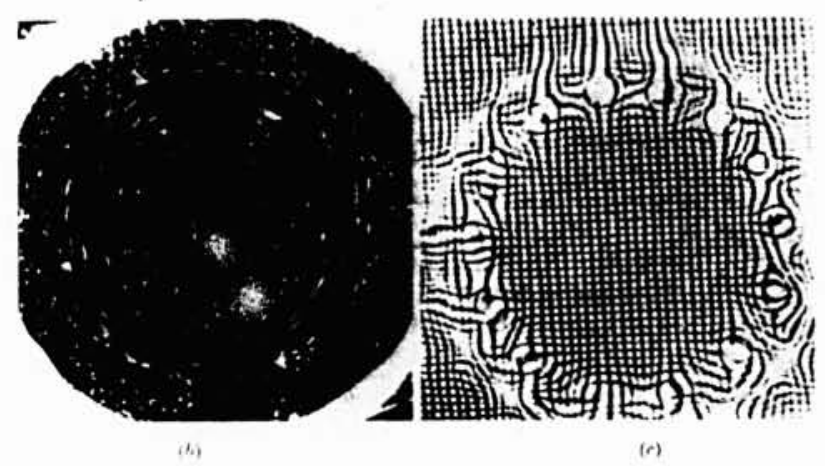

8. Expérience de Lehnert mettant en évidence la formation de colonnes alignées dans la direction du champ magnétique, $a$ : dispositif expérimental $(A$ et $D$, portions fixes du disque de cuivre ; $C$ : anneau de cuivre tournant); $b$ : photographie de la surface libre où les traits blancs représentent les trajectoires de grains de sable ; $c$ : image d'une grille à maille carrée réfléchie par la surface libre de mercure. L'épaisseur de la couche de mercure est $2 \mathrm{~cm}$, le champ magnétique est $0,43 \mathrm{~T}$. 
rience de Lehnert ne permet de mettre en évidence leur influence que parce que la couche de mercure est assez mince $(2 \mathrm{~cm})$.

\section{Exemples de phénomènes MHD en présence d'un champ alternatif}

Il n'est pas possible, dans ce panorama, de ne pas évoquer la classe des phénomènes observables en présence d'un champ magnétique alternatif. Le paramètre nouveau est la fréquence. Et son premier effet, bien connu, consiste à limiter la pénétration du flux magnétique dans le milieu conducteur. Dans les métaux l'épaisseur de peau $(2 / \mu \sigma \omega)^{-1 / 2}(\omega$ est la pulsation) est de l'ordre de $10 \mathrm{~cm}$ à $50 \mathrm{~Hz}$ et $1 \mathrm{~cm}$ à $5000 \mathrm{~Hz}$, elle devient si faible à des fréquences de l'ordre de $10^{5} \mathrm{~Hz}$ que l'on utilise alors une approximation de profondeur de pénétration nulle.

Dans cette peau électromagnétique, des courants de Foucault et un champ magnétique sont présents simultanément, tous les deux tangents à la frontière, en première approximation, et perpendiculaires l'un à l'autre. La force de Laplace est donc perpendiculaire à la frontière. Puisque $\vec{j}$ et $\vec{B}$ sont tous les deux pulsants et déphasés de $\pi / 4$, la moyenne de leur produit est non nulle. Dès que la fréquence est assez élevée (au-dessus de $20 \mathrm{~Hz}$ dans les métaux), le fluide ne ressent pratiquement plus que la valeur moyenne de cette force, et celle-ci se ramène essentiellement à une pression $B_{0}^{2} / 2 \mu\left(B_{0}\right.$ désigne l'amplitude du champ magnétique à la frontière du milieu conducteur). Cette pression se manifeste, par exemple, par la forme en dôme de la surface libre d'un métal fondu dans un four à induction à creuset (fig. 9).

Cette force électromagnétique stationnaire peut aussi être mise en œuvre pour léviter des masses de métal, que celui-ci
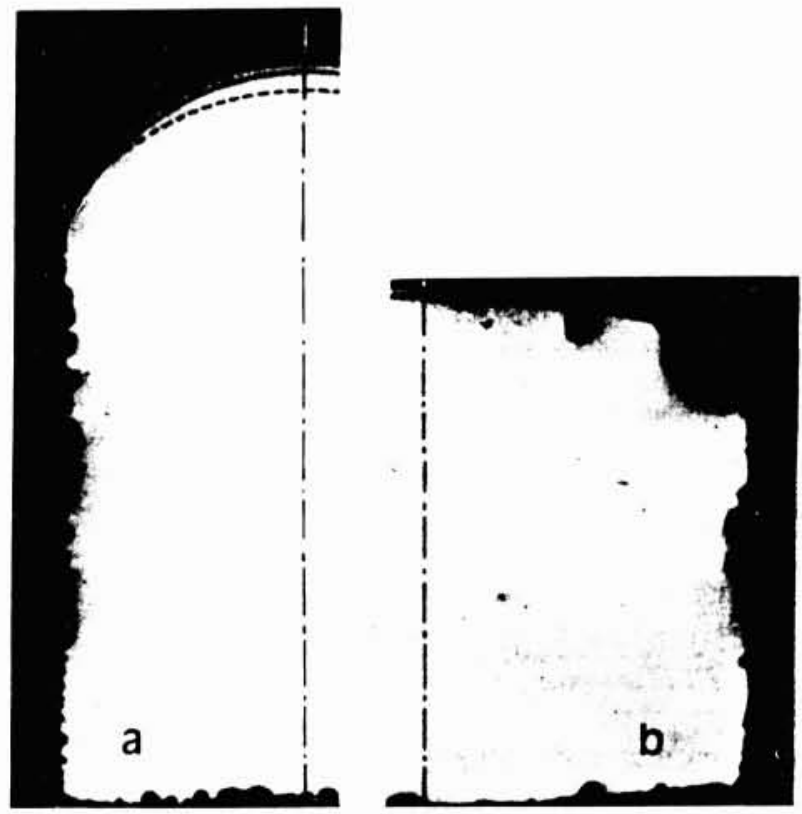

9. Coupes de deux lingots d'acier fondus dans un creuset à induction puis solidifiés par trempe brutale de façon à mettre en évidence la forme en dôme de la surface libre. Les traits continus et tiretés correspondent à deux approximations différentes de la surface libre. demeure à l'état solide, ou qu'il fonde sous l'effet de la dissipation par effet Joule, Certains ont ainsi pu rêver à des fours parfaits, où, sans pollution par le réfractaire puisque celui-ci n'existe plus, et sous atmosphère contrôlée, on pourrait élaborer des alliages extrêmement purs. Le système est illustré sur la figure 10. Ses limitations (la réalité n'est pas à la hauteur du rêve !) tiennent à l'axisymétrie qui impose au bas de la charge un point où, nécessairement, le champ magnétique et la force de Laplace sont nuls. Une sorte de goutte pendante se forme donc en ce point bas, portée par la tension superficielle contre la gravité. Les valeurs de la masse volumique et de la tension de surface des métaux fondus sont telles que l'on ne parvient pas à léviter des objets de dimensions supérieures à quelques $\mathrm{cm}$ pour l'aluminium et quelques $\mathrm{mm}$ pour le plomb.

Dans toutes ces configurations, four à creuset ou lévitation, on remarquera que, puisque $B_{0}$ varie le long de la frontière, la pression magnétique varie elle aussi. Ceci provoque un brassage de fluide, illustré sur la figure // dans

\section{métal fondu lévité}

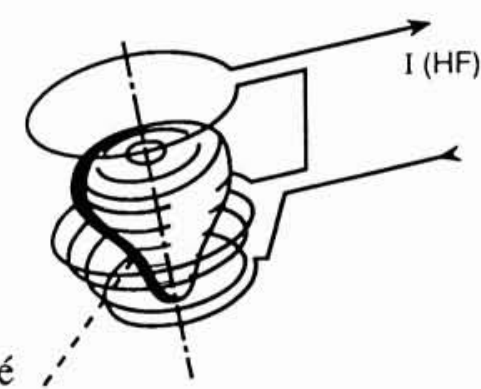

10. Schéma d'un dispositif de lévitation électromagnétique. Les spires inférieures assurent la poussée verticale, la spire supérieure assure la stabilité de la charge.

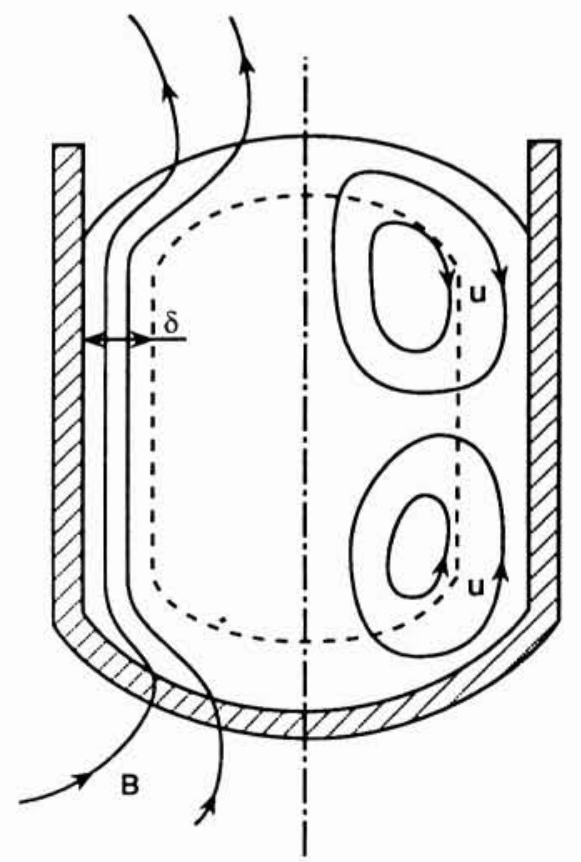

11. Schéma typique d'un creuset à induction centré dans un inducteur monophasé (non représenté). Le symbole $\delta$ représente l'épaisseur de peau électromagnétique où sont canalisées les lignes de flux magnétique. 
le cas d'un four à creuset. La circulation du fluide prend la forme de deux grands vortex toriques, le sens du mouvement allant, le long de la frontière, des régions où $B_{0}$ est faible (le voisinage de l'axe) vers les régions où $B_{0}$ est le plus grand (au milieu de l'inducteur).

\section{Stabilité et turbulence}

La présence d'une dissipation ohmique assez forte entraîne bien sûr des effets généralement stabilisants, mais on verra que ceci n'est cependant pas une règle générale. Le cas le plus clair, sans doute le premier à avoir fait l'objet d'études approfondies, est celui d'une couche fluide chauffée par dessous (le problème de Rayleigh-Bénard), en présence d'un champ magnétique uniforme colinéaire à la gravité. En l'absence d'un champ magnétique, le seuil à partir duquel apparaissent les rouleaux de Bénard est tel que $R a_{\text {crit }}=$ 1708 , où :

$$
R a=\frac{g \beta \Delta T h^{3}}{v \alpha}
$$

désigne le nombre de Rayleigh ( $\beta$ est la dilatibilité, $\Delta T$ la différence de température imposée, $h$ l'épaisseur de la couche fluide et $\alpha$ la diffusivité thermique). En présence d'un champ magnétique assez fort, caractérisé par le nombre de Hartmann (7), le nombre de Rayleigh critique s'accroît très vite, comme $\mathrm{Ha}^{2}$. Et, parallèlement, la largeur des rouleaux, qui est du même ordre de grandeur que $h$ à champ magnétique nul, devient beaucoup plus petite ; elle varie comme $\mathrm{Ha}^{-1 / 3}$.

Autre exemple d'effet stabilisant, lorsqu 'un champ magnétique alternatif est appliqué parallèlement à une surface libre de métal fondu, les éventuelles déformations de la surface peuvent être stabilisées [5], mais le mécanisme est tout à fait différent de celui discuté ci-dessus. Il n'est pas dû à la dissipation ohmique associée à un mouvement du fluide (les déformations de la surface pourraient être immobiles), mais à la pression magnétique $B_{0}^{2} / 2 \mu$, plus grande au droit de bosses (qui interceptent des lignes de flux magnétique) que dans les creux. La surpression magnétique associée aux bosses a tout simplement pour effet de les repousser, comme une force de rappel élastique. On notera cependant que cet effet stabilisant s'annule exactement si le vecteur d'onde des perturbations est perpendiculaire au champ magnétique, puisque, dans ce cas, les bosses n'interceptent aucune ligne de flux.

Il serait cependant dangereux de croire que les effets MHD sont toujours stabilisants. L'exemple le plus fameux de situations instables est certainement celui des jets parcourus par un courant électrique. Ce courant réagit avec son propre champ magnétique pour exciter des perturbations du jet. La figure 12 montre les deux modes visibles, le mode dit « en saucisses " observable lorsque le seul champ magnétique est le champ propre du courant passant dans le jet, et le mode " en spirales" observable lorsque l'on rajoute un champ magnétique axial suffisant.

La turbulence des écoulements de métaux liquides est, elle aussi, fortement modifiée en présence d'un champ magnétique. La présence de la dissipation par effet Joule associée au courant induit par les fluctuations de vitesse entraîne, certes, une réduction du niveau de turbulence. Mais cela s'avère beaucoup plus subtil...

On observe effectivement que, si $\mathrm{Ha} / \mathrm{Re}$ est supérieur à une valeur critique voisine de $2,510^{-2}$, la loi expérimentale

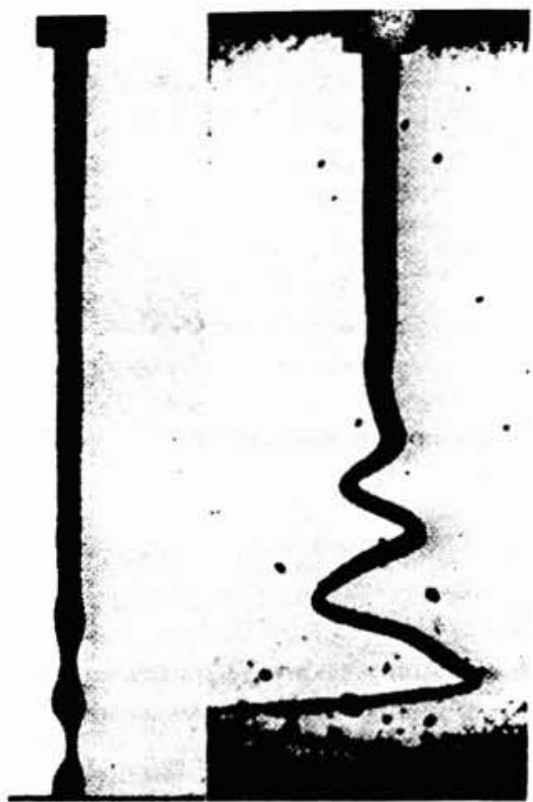

12. Instabilité de jets de mercure verticaux parcourus par un courant électrique continu, à gauche sans champ magnétique autre que celui du courant, à droite avec un champ magnétique axial superposé.

de perte de charge coïncide avec la loi laminaire. Peut-on en déduire, comme les premiers chercheurs qui ont observé ce résultat, que l'écoulement a été laminarisé ? Pas du tout ! Dès que l'on fut capable d'introduire des sondes sensibles à la turbulence dans ces écoulements, la présence de turbulence fut bien mise en évidence, même lorsque $\mathrm{Ha} / \mathrm{Re} \gg 2,510^{-2}$. Des études très approfondies ont suivi ces premières découvertes et la dynamique originale de cette turbulence MHD est maintenant assez bien comprise.

L'explication de l'apparente laminarisation tient au fait que la turbulence devient bidimensionnelle, les tourbillons devenant des colonnes alignées dans la direction du champ magnétique. Le champ de vitesse bidimensionnel a la propriété curieuse de n'induire aucun courant et, par suite, de ne subir aucune dissipation ohmique. Et la turbulence bidimensionnelle a aussi la propriété singulière de ne subir qu'une très faible dissipation visqueuse. Ceci est dû à l'inversion du processus de cascade : à deux dimensions les tourbillons s'associent, en forment de plus gros, et ainsi de suite. Le flux d'énergie n'est donc plus dirigé, comme à trois dimensions, vers des échelles assez petites pour dissiper l'énergie par viscosité, il est au contraire dirigé vers des échelles de plus en plus grandes, insensibles à la viscosité, sauf aux frontières du domaine fluide. Cette turbulence MHD rendue bidimensionnelle parvient donc à échapper aux deux formes de dissipation qui la guettent. Elle survit donc sans prélever d'énergie sur l'écoulement moyen qui la transporte, et c'est pourquoi la perte de charge de celui-ci obéit à une loi laminaire.

Mais pourquoi la turbulence MHD bidimensionnelle n'induit-elle pas de courant? Imaginons, dans un plan perpendiculaire au champ magnétique $\vec{B}$ uniforme, un contour matériel fermé $C$. La turbulence le déforme, mais en raison de l'incompressibilité elle ne change pas l'aire limitée 
par ce contour. Par ailleurs, $\vec{B}$ est uniforme. Ce contour $C$ n'est donc soumis à aucune variation de flux magnétique. Conformément à la loi de Lenz, il n'est soumis à aucun effet d'induction, il n'est parcouru par aucun courant électrique. Ce raisonnement s'applique à tout contour $C$. Tant que la turbulence demeure bien bidimensionnelle, elle n'induit donc aucun courant électrique et ne subit aucune dissipation par effet Joule.

Reste à comprendre pourquoi la turbulence devient bidimensionnelle. Cela tient à un double mécanisme. En premier lieu, les ondes d'Alfven allongent tous les tourbillons dans la direction de $\vec{B}$. Pour que ceci soit efficace, il faut que les parois perpendiculaires à $\vec{B}$ soient assez proches, de telle sorte que l'atténuation des ondes soit négligeable. En second lieu, il faut que les parois soient isolantes. Ceci force les extrémités des tourbillons à être bien perpendiculaires aux parois, sinon il faudrait qu'un courant électrique pénètre dans la paroi. Des expériences réalisées à Grenoble [6] (voir fig. 13) ont permis de bien valider ces idées, appuyées par ailleurs sur une théorie solide [7].

\section{Quelques remarques en guise de conclusion}

Quelle richesse dans ce couplage entre les phénomènes hydrodynamiques et électromagnétiques! Ces quelques pages n'en donnent qu'un bref aperçu. Elles laissent entièrement de côté les gaz ionisés, qui introduiraient la compressibilité (alors les ondes d'Alfven interagissent avec les ondes acoustiques). Et il était exclu d'engager ici, dans un texte aussi court, une réflexion sur les applications actuelles ou potentielles de la MHD. Le lecteur aura cependant noté, à travers des exemples cités, comme le four à induction, que ces applications sont tout à fait réelles, notamment en génie métallurgique.

Cette petite excursion à travers des phénomènes MHD laissera peut-être au lecteur non initié une impression de complexité. Au contraire, les spécialistes jugeront sans doute sommaires les raisonnements raccourcis utilisés pour éviter les théories approfondies. Il était en réalité difficile de donner en quelques pages, donc sans explications détaillées, à la fois un panorama de quelques phénomènes MHD et une première interprétation de chacun d'eux. Pour l'auteur, deux qualificatifs s'imposent pour caractériser cette MHD par comparaison avec la mécanique des fluides classique: subtile et spectaculaire.
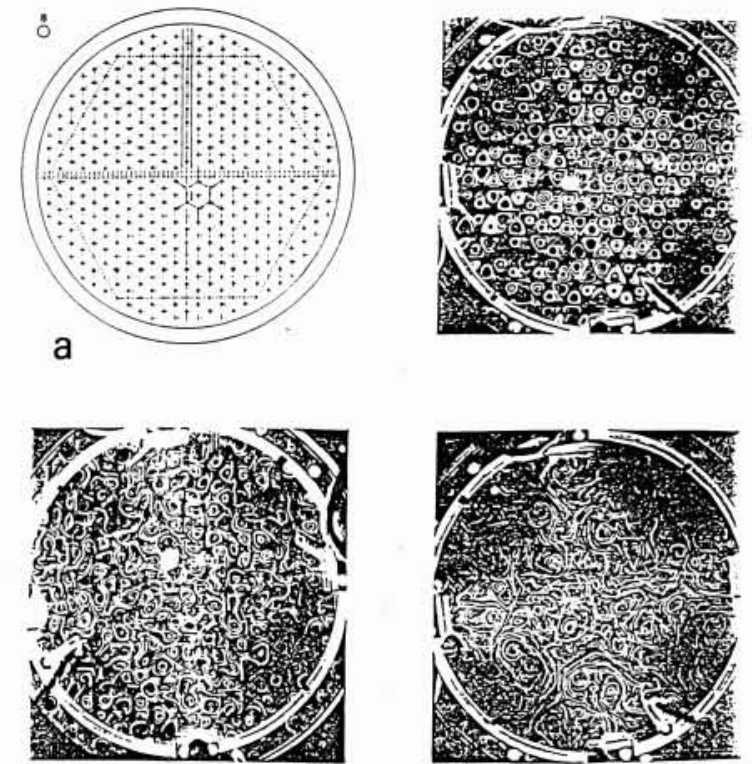

C

d

13. Expérience de Somméria mettant en évidence la cascade inverse dans une couche de mercure horizontale ( $2 \mathrm{~cm}$ d'épaisseur) en présence d'un champ magnétique vertical uniforme de $0,3 \mathrm{~T}$ ( $a$ : réseau hexagonal d'électrodes permettant d'établir le mouvement périodique visible en $b$ lorsque le courant devient assez élevé pour rendre instable et turbulent le réseau de tourbillons initialement périodiques).

\section{Références}

[1] MOREAU R., 1990. - Magnetohydrodynamics. Kluwer Acad. Pub.

[2] Alfven H. and Fälthammar C.G., 1963. - Cosmical Electrodynamics. 2nd ed., Oxford Univ, Press.

[3] Lehnert B., 1955. - Proc. Roy. Soc. London A 233, p. 299.

[4] Chandrasekhar S., 1961. - Hydrodynamic and Hydromagnetic Stability. Oxford Univ. Press.

[5] Garnier M. et Moreau R., 1983. - J. Fluid Mech., vol. 127 , p. 365.

[6] Somméria J., 1986. - J. Fluid Mech., vol. 170, p. 139.

[7] Somméria J. el Moreau R., 1982. - J. Fluid Mech., vol. 118, p. 507. 